\title{
Lebenserwartung bei klinisch diagnostizierter Demenz
}

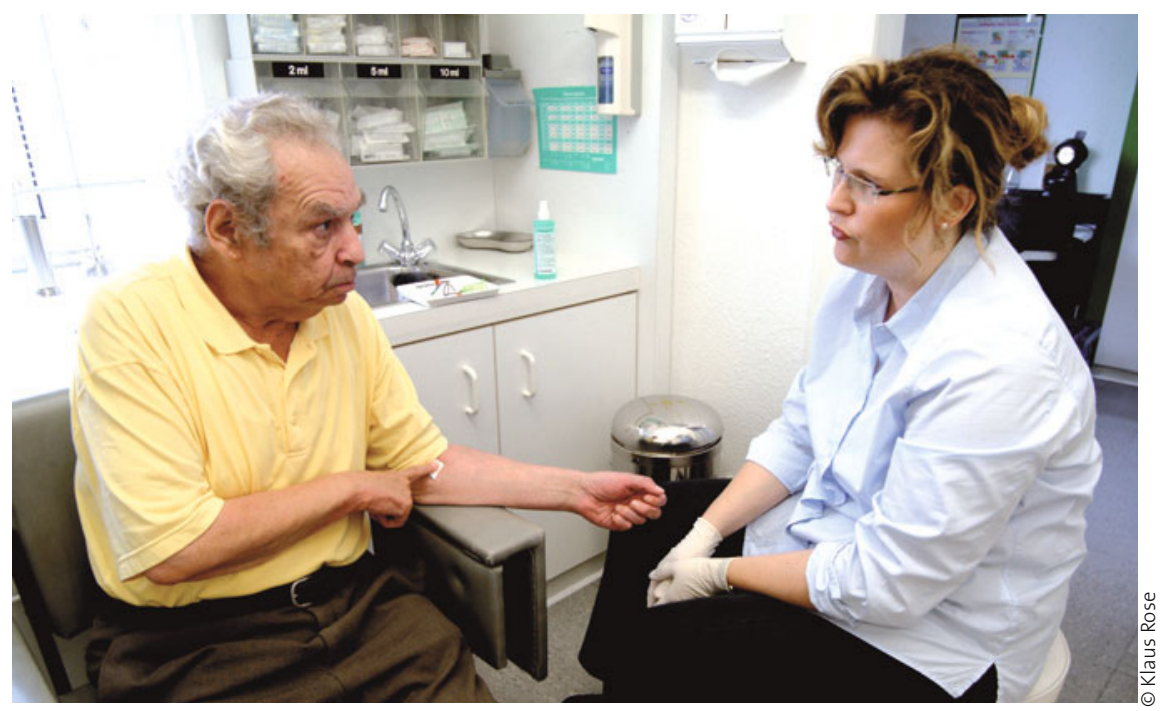

Mehr als die Hälfte der Patienten mit fortgeschrittener Demenz sterben innerhalb von sechs Monaten.

In einer englischen Studie untersuchten Forscher Patienten aus 353 Allgemeinpraxen, bei denen zwischen 1990 und 2007 die Diagnose einer Demenz gestellt wurde. Es fanden sich über 22500 solcher Patienten, die in der Folge mit einer Kontrollgruppe aus altersgleichen nicht Dementen verglichen wurde.

- Die Demenzinzidenz stiegt erwartungsgemäß mit zunehmendem Alter steil an und betrug bei den > 90-jährigen das 15 -Fache im Vergleich mit 60-69-jährigen. Die mittlere Überlebenszeit nach Diagnosestellung betrug bei den 60-69-jährigen 6,7 Jahre, bei den > 90-jährigen 1,9 Jahre. Die Mortalität in der Demenzgruppe lag über die gesamte Beobachtungszeit etwa um das 3-Fache höher als bei den nicht Dementen.

\section{Kommentar}

Es überrascht nicht, dass die Überlebenszeit nach klinisch diagnostizierter Demenz kürzer ist als bei Patienten, bei denen die Diagnose mittels psychometrischer Tests im Rahmen von Screeninguntersuchungen gestellt wurde. Dennoch ist es wieder einmal eine eindrucksvolle Studie, die uns zeigt, welch erheblich lebensverkürzenden Effekt eine Demenz besonders beim Hochbetagten hat. Dies ist wichtig, z.B. wenn es darum geht zu entscheiden, welche Maßnahmen bei einem Demenzkranken noch angemessen und sinnvoll sind und welche besser unterbleiben sollten. Man weiß, dass Angehörige und Ärzte, die nicht über die hohe Mortalität bei Demenz aufgeklärt sind, eher dazu neigen, pro Krankenhauseinweisung, pro Intensivstation, pro Beatmung oder PEG etc. zu votieren. Dies, obwohl mehr als die Hälfte der Patienten mit fortgeschrittener Demenz innerhalb von sechs Monaten sterben. „Fortgeschritten “ bedeutet, dass diese Menschen Hilfe bei den einfachen Alltagsverrichtungen benötigen und ihre Angehörigen häufig nicht mehr erkennen. In diesem Krankheitsstadium sollten daher behutsame, nach palliativmedizinischen Gesichtspunkten maßgeschneiderte Behandlungskonzepte zum Einsatz kommen.

J. Zeen $~-$

\section{- G. Rait et al.}

Survival of people with clinical diagnosis of dementia in primary care: cohort study. Brit. Med. J. 341: doi:10.1136/bmj.c3584 